\title{
Reflets
}

Revue ontaroise d'intervention sociale et communautaire

\section{Les Sourdes et la communication}

\section{Monique Boudreault}

Volume 3, numéro 2, automne 1997

Visibles et Partenaires : Pratiques et recherches féministes

URI : https://id.erudit.org/iderudit/026184ar

DOI : https://doi.org/10.7202/026184ar

Aller au sommaire du numéro

Éditeur(s)

Reflets : Revue ontaroise d'intervention sociale et communautaire

ISSN

1203-4576 (imprimé)

1712-8498 (numérique)

Découvrir la revue

Citer cet article

Boudreault, M. (1997). Les Sourdes et la communication. Reflets, 3(2), 242-249.

https://doi.org/10.7202/026184ar

Tous droits réservés (C) Reflets : Revue ontaroise d'intervention sociale et communautaire, 1997

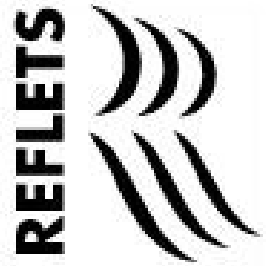

Ce document est protégé par la loi sur le droit d'auteur. L’utilisation des services d’Érudit (y compris la reproduction) est assujettie à sa politique d'utilisation que vous pouvez consulter en ligne.

https://apropos.erudit.org/fr/usagers/politique-dutilisation/ 


\title{
Les Sourdes et la communication
}

\begin{abstract}
Monique Boudreault
Représentante de la communauté des femmes sourdes et professeure au Collège Boréal, Sudbury ${ }^{1}$
\end{abstract}

Je suis Sourde ${ }^{2}$ de naissance. Mes parents ont eu sept enfants dont trois sont Sourds. Je suis allée au couvent des soeurs de la Charité de la Providence à l'Institution des Sourdes-Muettes ${ }^{3}$ de Montréal. Je me suis mariée avec un Sourd et j'ai deux garçons. L'un d'eux est Sourd; il a 26 ans et l'autre en a 23. Dans ma parenté, il y a huit Sourds. Dans ma carrière professionnelle, trois étapes sont importantes. J'ai été d'abord fonctionnaire pour la province de Québec pendant 11 ans, puis éducatrice spécialisée (enseignant aux adultes sourds à devenir autonomes dans la vie quotidienne) pendant dix ans. Depuis 1992, je suis professeure dans le programme de communication en langue des signes au Collège Boréal.

Au cours de ma vie, j'ai participé à divers organismes desservant les Sourds et les personnes qui ne le sont pas. Je suis actuellement vice-présidente du Collectif des femmes francophones du Nord-Est de l'Ontario. C'est d'ailleurs à leur invitation que j'ai participé au colloque Visibles et Partenaires. En effet, lors des réunions du Collectif, les membres ont dû apprendre à communiquer avec une Sourde et à travailler avec l'aide d'interprètes en langue des signes. Suite à leurs efforts, à leur flexibilité et à leurs nouvelles connaissances de la culture sourde, elles ont voulu que j'en parle à un plus large public afin que toutes soient davantage sensibilisées aux difficultés que rencontrent quotidiennement les Sourdes et les Sourds. Lors du colloque, j'ai été heureuse de faire une présentation en langue des signes pour mieux faire connaître et comprendre la réalité des femmes sourdes dans des situations de communication quotidienne. 
Dans cet article, je discuterai brièvement du handicap qu'est la surdité avant de présenter quatre situations vécues par les Sourdes, comme par les Sourds, notamment dans le domaine de l'éducation. En conclusion, je regarderai certains éléments à mettre en place pour améliorer la qualité de vie des Sourdes et des Sourds.

\section{Le handicap}

Vous voyez tous les jours des handicapés physiques, des aveugles ou des handicapés mentaux. Pour les Sourds, le handicap est différent car la surdité est invisible, elle n'est pas apparente. Il est alors très difficile de deviner qu'une personne est sourde. Mais, la vie quotidienne des Sourdes et des Sourds n'est pas facile à cause du manque d'accessibilité des services (interprétation, communication, refus d'emploi à cause de la surdité, etc.).

Par exemple, après une agression sexuelle, les Sourdes doivent affronter des obstacles majeurs au chapitre de la communication. Le personnel du centre d'aide auquel elles pourraient s'adresser et qui, en principe, devrait leur offrir des services, ne peut pas communiquer avec elles. La plupart du temps, il ne connaît même pas le service d'interprétation. Comment une Sourde peut-elle alors recevoir ce service? La même situation se produit lorsque la police procède à l'arrestation d'une Sourde et qu'elle lui menotte les deux mains, comment cette personne peut-elle communiquer? Comment une Sourde peut-elle demander les raisons de son arrestation ou l'aide d'un avocat? Cela lui est impossible car elle ne peut ni parler ni écrire. C'est encore la même situation à l'urgence d'un hôpital où il est impossible de recevoir les services d'interprétation en langue des signes et où aucun employé ne connait la langue des signes.

Les Sourdes ont aussi de la difficulté à obtenir un emploi à cause de la discrimination qui s'exerce contre elles et ce malgré les politiques pour favoriser l'intégration des handicapés, notamment les Sourdes. Peu de services leurs sont offerts. 
La vie des Sourdes et des Sourds est difficile car même si elles peuvent fonctionner normalement, elles font face à de la discrimination à cause de l'incapacité mutuelle de communication. Je crois que la discrimination envers ces personnes est très répandue. Ironiquement, je dirais que la meilleure chose qui puisse arriver à la communauté sourde serait de vivre sur «une planète de Sourds» ${ }^{4}$.

\section{Difficultés quotidiennes des Sourdes}

J'aimerais brièvement présenter quelques-unes des situations difficiles qui parsèment la vie quotidienne des Sourdes et des Sourds et offrir quelques pistes afin d'améliorer leur qualité de vie.

\section{L'enfance}

Dans l'enfance, faute de moyen de communication, plusieurs Sourds sont protégés par leurs parents, tandis que d'autres sont rejetés. La plupart des parents n'acceptent pas que leurs enfants utilisent la langue des signes car ils trouvent que c'est «pas beau à voir», «trop difficile à apprendre» ou «ils ont honte de montrer que leur enfant est sourd».

Lors d'une intervention, effectuée en 1995 auprès des Sourdes, voici ce qu'une femme me disait:

Moi, je n'aimais pas faire des visites dans la famille. Je ne voulais pas y aller, mais mes parents m'obligeaient. Je restais assise à côté de ma mère et regardais le monde parler. Je demandais souvent à maman de quoi elle parlait avec les autres. Elle me répondait toujours la même chose: «reste tranquille, je vais t'expliquer tantôt». Mais finalement, elle ne m'expliquait jamais rien. J'avais manqué toute la discussion, c'était pas drôle mais ça arrivait souvent. 
Comment les jeunes Sourds peuvent-ils trouver une satisfaction dans la vie quotidienne? Pour eux, la langue des signes est un moyen de communication, malgré qu'il soit difficile pour les parents d'apprendre cette langue. Pourtant, c'est encourageant pour les jeunes Sourds lorsqu'enfin ils peuvent communiquer avec leurs parents, leurs frères et soeurs, etc. C'est important pour eux de pouvoir dialoguer comme n'importe quel être humain.

\section{L'éducation}

De l'Âge de pierre jusqu'aux temps modernes (avant 1760), il n'y avait presque rien sur l'histoire des Sourds. Ces derniers communiquaient avec des gestes et du mime pour se faire comprendre des entendants et ils vivaient dans l'isolement. En 1760, l'Abbé de l'Épée ouvrit la première école publique des Sourds à Paris. Cette institution a marqué la création des multicultures sourdes dans le monde. Au Canada, la première institution des Sourds-Muets a été fondée en 1831 et a été dirigée par Ronald MacDonald. Elle était située à Québec. Plusieurs langues ont alors été utilisées dans l'éducation des Sourds-Muets: le vieux ASL (American Signs of the Language), le français et l'anglais. Le professeur sourd Antoine Caron a soutenu MacDonald et l'édifice où se trouvait l'école existe encore non loin de la Porte SaintJean à Québec. Ce n'est qu'en 1851 que la communauté des Soeurs de la Charité de la Providence a fondé la première Institution des Sourdes-Muettes de Montréal. Auparavant, garçons et filles fréquentaient les mêmes institutions. En 1880, l'utilisation de la langue des signes est interdite dans l'enseignement par les participants entendants du Congrès de Milan, présidé par le Père Tarra. Quelques années plus tard, le célèbre inventeur du téléphone, Abraham Graham Bell, prône l'abolition de la langues des signes dans l'enseignement. Et vers 1900, on assiste, aux États-Unis, à la fermeture des écoles spécialisées dont $40 \%$ des professeurs étaient Sourds. Avec l'arrivée de la Révolution tranquille, dans les années 1960, le gouvernement du Québec ferme malheureusement les écoles pour les Sourds. Ces fermetures entraîneront des différents entres les diverses communautés pour le choix de la 
meilleure langue d'enseignement et ce sont les Sourds qui en subiront les conséquences comme en fait foi ce témoignage.

En 1981, quand j'avais 11 ans, j'ai été renvoyée de l'école des Sourds. Le professeur exigeait que j'utilise le français signé ${ }^{5}$ conformément à la nouvelle politique de l'établissement. Les enseignants me disaient que la langue des signes de mes parents Sourds n'était pas valable. Je me suis sentie humiliée et dévalorisée, car la LSQ (langue des signes du Québec) est ma source d'inspiration et ma soif d'apprendre (extrait de la revue Entendre, mars 1997, p. 8).

Faute d'enseignement dans leur langue (langue des signes), les Sourdes et les Sourds ont été forcés de s'intégrer dans les écoles des entendants après les fermetures de leurs institutions en 1970. Auparavant, les femmes avaient la chance de suivre des cours réguliers dans leur langue. Par exemple, à l'Institut familial, les femmes recevaient une éducation de base tout en apprenant à prendre soin de la famille, à faire la cuisine ou à coudre, etc. Après la fermeture de ces institutions, la plupart des étudiants n'ont pas continué leurs études collégiales. Faute d'une meilleure intégration dans les institutions scolaires largement réservées aux entendants, nombreux sont ceux et celles qui abandonnent l'école après le niveau primaire ou secondaire et ces personnes vivent souvent isolées après des essais de communication infructueux.

C'est pourquoi, et ce malgré les changements dans le système scolaire, je conseille fortement aux parents d'enfants sourds, aux professeurs sourds de la LSQ, aux interprètes de la LSQ et aux professionnels de trouver ensemble de bonnes solutions pour satisfaire les jeunes en ce qui a trait à leur avenir scolaire et à leur intégration sur le marché du travail.

Par ailleurs, la LSQ n'empêche nullement les jeunes d'apprendre à parler, à lire et à écrire en français. Au contraire, le schéma présenté ci-après est un modèle que je recommande pour faciliter l'apprentissage des langues chez les jeunes Sourdes et Sourds. 
- Apprendre la LSQ comme langue de communication.

- Apprendre le français comme langue seconde, enseignée au moyen de la LSQ.

- Apprendre à parler si l'enfant le peut et est motivé à le faire.

- Apprendre d'autres langues des signes ou d'autres langues orales en mettant l'accent sur l'écriture et la lecture.

- Apprendre une ou plusieurs autres langues parlées si tel est le désir de l'enfant.

\section{La culture sourde}

Les Sourdes, sur la base de leur identité, ont aussi intégré une culture sourde. Cette culture se rapporte à leurs valeurs longtemps niées. Ce ne sera que vers les années 1980, parallèlement au mouvement féministe, qu'apparaîtra la révolution de la culture sourde. Suite au congrès de Hambourg en Allemagne et 110 ans après le congrès de Milan, on assiste alors à un retour de la reconnaissance des langues des signes dans le monde.

Saviez-vous que le Canada est le champion en ce qui a trait aux droits de la personne pour le multiculturalisme, sauf pour notre culture sourde? On tente surtout de nous inculquer des valeurs d'entendants alors que l'on ne reconnait pas nos propres valeurs et notre propre culture sourde. Ce qu'on essaie encore d'abolir, c'est la valeur de notre moyen de communication, c'està-dire la langue des signes.

Afin de reconnaitre la culture sourde, les gouvernements, les parents des enfants sourds, les professionnels, etc., devraient travailler avec nous, les Sourds, et non nous imposer des valeurs qui ne nous conviennent pas. De plus, nous voudrions que toutes les instances reconnaissent notre langue.

\section{La communication}

La communication est souvent source de frustrations chez les Sourds et constitue la principale barrière à leur fonctionnement social.

Avant 1980, on utilisait les services d'interprétation bénévole, tout particulièrement ceux des parents ou de la famille pour les 
visites médicales par exemple. Après 1980, les interprètes offrent leurs services partout (médecins, écoles, etc.), mais leur service est souvent de mauvaise qualité à cause de leur manque d'expérience. Depuis dix ans, la technologie offerte pour les Sourds accuse des progrès remarquables. Par exemple, pour téléphoner, les femmes peuvent maintenant utiliser la langue des signes avec un service d'interprétation ou à l'aide des services de relais Bell $(711)^{6}$, d'urgence (911) ou communiquer directement entre elles. Toutefois, les services téléphoniques de relais ou d'urgence sont souvent médiocres malgré les critiques et les protestations faites envers la compagnie Bell. Suite à ces critiques, on ne voit toujours aucune amélioration. Je me demande parfois si la compagnie Bell ne travaille pas seulement pour de l'argent au détriment de la qualité du service offert aux Sourds.

Pour pallier aux problèmes de communication, les parents d'enfants sourds, les parents sourds, les enfants sourds, les professeurs sourds de la LSQ, les professeurs entendants en français, les interprètes sourds et entendants de la LSQ et les professionnels devront former un nouveau regroupement pour uniformiser la langue des signes et briser les obstacles quotidiens vécus par les Sourdes et les Sourds.

\section{Conclusion}

Afin que soit reconnue la langue des signes, nous devons veiller à ce que l'enseignement de la langue des signes soit dirigé par les Sourds. Ceux qui connaissent la communauté sourde saisissent généralement bien leurs besoins linguistiques et culturels. La LSQ, comme d'ailleurs l'ASL (American Signs of the Language), doit être reconnue dans tous les programmes comme l'une des langues officielles du Canada et comme l'une des langues d'origine pour le peuple Sourd. Nous devons aussi appuyer le droit du peuple Sourd à apprendre dans sa propre langue que ce soit en français ou en anglais. Il nous faut aussi valoriser la culture sourde et sa capacité d'analyser ses besoins en matière de programmes scolaires. 
Nous devons appuyer la nécessité d'éduquer les Sourds dans leur langue maternelle afin d'assurer la transition vers l'une des langues officielles du Canada. La communauté sourde souhaite que la communauté entendante appuie le grand changement de la qualité de la vie des Sourds par la communication quotidienne en langue des signes.

Soyons fiers d'être Sourds car, comme le dit George W. Verditz dans un film de l'Association des Sourds des États-Unis,

Tant qu'il aura des Sourds sur terre il existera des signes, et tant que nous aurons nos films [et j'ajoute tout le reste], nous pourrons préserver notre précieuse langue des signes dans sa pureté originale. Nous espérons que tous ensemble nous aimerons et protégerons notre belle langue des signes. Elle est le plus beau cadeau que Dieu ait donné aux Sourds.

\section{Notes}

1. NDLR:Cette communication a été présentée en LSQ avec le service d'une interprète. Le comité de rédaction a cherché à préserver son caractère original. L'auteure remercie Nathalie Dalpé pour son aide à la rédaction de cet article.

2. L'usage du «S» majuscule pour le nom ou parfois pour le qualificatif «Sourd» est une prise de position de l'auteure en faveur de la reconnaissance de la population des Sourdes et des Sourds et de leur identité culturelle.

3. Le terme Sourdes-Muettes n'est plus approprié. Les Sourds ne parlent pas car ils ne peuvent répéter les sons qu'ils n'entendent pas.

4. L'auteure fait ici référence à un livre très populaire sur la surdité: La planète des Sourds de Jean Grémion.

5. À la différence de la LSQ qui est une langue à part entière et ayant des critères qui lui sont propres, le français signé est un code. Il consiste à faire des signes sur la structure du français.

6. Pour téléphoner à une personne entendante, le client sourd compose le numéro du Service de relais Bell (711). Au moyen d'un téléimprimeur ou télétype (TTY) doté d'un écran d'affichage, le téléphoniste reste en ligne pour relayer fidèlement les messages par sa voix à une personne entendante. 